\title{
ANOC: Anonymous Network-Coding-Based Communication with Efficient Cooperation
}

\author{
Peng Zhang, Yixin Jiang, Chuang Lin, Patrick P.C. Lee, and John C.S. Lui
}

\begin{abstract}
Practical wireless network coding (e.g., COPE) is a promising technique that can enhance the throughput of wireless networks. However, such a technique also bears a serious security drawback: it breaks the current privacy-preserving protocols (e.g., Onion Routing), since their operations conflict each other. As user privacy in wireless networks is highly valued nowadays, a new privacy-preserving scheme that can function with wireless network coding becomes indispensable.

To address such a challenge, we apply the idea of cooperative networking and design a novel anonymity scheme named ANOC, which can function in network-coding-based wireless mesh networks. ANOC is built upon the classic Onion Routing protocol, and resolves its conflict with network coding by introducing efficient cooperation among relay nodes. Using ANOC, we can perform network coding to achieve a higher throughput, while still preserving user privacy in wireless mesh networks. We formally show how ANOC achieves the property of relationship anonymity, and conduct extensive experiments via nsclick to demonstrates its feasibility and efficiency when integrated with network coding.
\end{abstract}

Index Terms-Network coding, anonymity, cooperative networking, Onion Routing.

\section{INTRODUCTION}

How to achieve high data throughput is a critical concern in wireless networks. Recent studies show that network coding [1], as an alternative to the traditional store-and-forward paradigm, can remarkably enhance the network capacity. In particular, authors in [2] propose COPE, the first practical wireless network coding scheme for wireless mesh networks [3]. In COPE, nodes operate in promiscuous mode, and opportunistically perform data mixing (or coding) on the packets to be forwarded to neighboring nodes. Fig. 1 shows three basic coding scenarios in COPE [4]. In Fig. 1(a), node $S_{1}$ needs to send a packet $P_{1}$ to $D_{1}$, and this packet is relayed by node $C$; while $S_{2}$ needs to send a packet $P_{2}$ to $D_{2}$, also relayed by node $C$. The dashed line means that $D_{1}$ and $D_{2}$ can overhear $P_{2}$ and $P_{1}$, respectively, due to the broadcast nature of wireless channels. Without network coding, the communication will cost four transmissions in total: (1) $S_{1}$ sends $P_{1}$ to $C$, (2) $C$ forwards $P_{1}$ to $D_{1}$, (3) $S_{2}$ sends $P_{2}$ to $C$, and (4) $C$ forwards $P_{2}$ to $D_{2}$. On the other hand, with network coding, the relay node $C$ only needs to broadcast $P_{1} \oplus P_{2}$, and then $D_{1}$ can recover $P_{1}$ by computing $P_{2} \oplus\left(P_{1} \oplus P_{2}\right) ; D_{2}$ can recover $P_{2}$ by computing $P_{1} \oplus\left(P_{1} \oplus P_{2}\right)$. In this way, one transmission will be saved at node $C$, and the network throughput can be improved. Fig. 1(b) shows another possible coding scenario where no overhearing is needed; Fig. 1(c) gives a hybrid scenario that combines the former two cases.

In addition to throughput improvement, privacy preservation is also an important concern in wireless communications since:

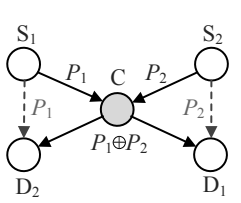

(a) Coding scenario with overhearing

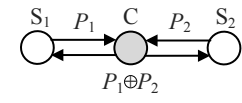

(b) Coding scenario without overhearing

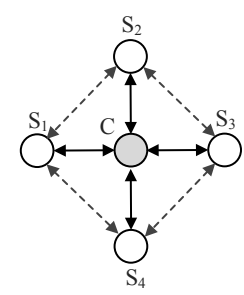

(c) Hybrid coding scenario
Fig. 1: Basic coding scenarios of COPE [4].

(1) online privacy is highly valued by wireless users nowadays; and (2) the open-air traffic in wireless medium can be easily monitored and traced. Consider for example, a scenario where multiple clients can access a server $S$ via a wireless mesh network. Equipped with targeted antennas, an adversary can easily intercept traffic by staying close to server $S$, and then perform traffic analysis [5] so as to deduce the identities of users who have accessed $S$. Depending on the specific service provided by $S$, sensitive information, such as "who has accessed a web page or downloaded a file", will be disclosed. It is important to note that end-to-end encryption (e.g., SSL/TLS) only provides a limited form of privacy: while end-to-end encryption hides application payload from the adversary, the adversary can still learn the IP addresses of the client and the server in a data session.

Many techniques are proposed to provide user privacy in communication networks: Mix-Net [6]-[8], Onion Routing [9]-[11], and Crowds [12] are shown to be effective in wired networks; ANODR [13], WAR [14], and Onion Ring [15] are more suitable for wireless applications. However, when a wireless network is upgraded to enable network coding, many of the above privacy-preserving protocols will not be applicable. The core reason is that the packet-mixing operations required by network coding are in conflict with the encryption/decryption operations required by the privacypreserving schemes at relay nodes (details will be given in Section III). Considering the rising privacy concern, as well as the increasing bandwidth demand in wireless networks, an efficient privacy-preserving scheme that can work with wireless network coding becomes highly important.

To address the above issue, this paper proposes ANOC, i.e.,

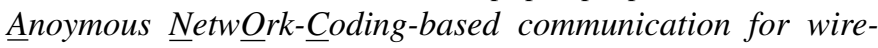
less mesh networks. ANOC uses Onion Routing as its building block, and resolves the conflict between Onion Routing and network coding by introducing efficient cooperation (sessionkey sharing and auxiliary decrypting) among relay nodes. 
Specifically, we mainly address the following two challenges: (i) how to trigger the session-key sharing in an on-demand fashion, and (ii) how to efficiently and securely share session keys with neighbors without leaking any information to adversaries.

With these challenges addressed, we formally show that ANOC can achieve a practical privacy requirement called relationship anonymity (i.e., unlinkability [16]), meaning that adversaries cannot associate any sender with the corresponding receiver of a data session by simply observing the wireless traffic. We also conduct extensive experiments via nsclick [17] to show that ANOC can work efficiently with network coding in wireless mesh networks.

In summary, our contribution is two-fold: 1) to the best of our knowledge, this is the first paper to address the privacy vulnerability of wireless network coding; 2) we propose, implement, and evaluate a novel anonymous communication scheme for network-coding-based wireless mesh networks, using techniques of cooperative networking.

The remainder of this paper is organized as follows. Section II surveys some related work. Section III gives a formal statement of the problem to be studied. Section IV motivates the basic idea of ANOC, the implementation of which is detailed in Section V. Section VI and Section VII present analytical and experimental results, respectively. Finally, Section VIII concludes the paper.

\section{RELATED WORK}

Security and privacy issues in random linear network coding have attracted much attention. Potential threats such as pollution attacks, eavesdropping attacks, traffic analysis attacks, entropy attacks are treated in [18]-[21], respectively. However, for the wireless network coding paradigm, e.g., COPE, little attention have been paid to addressing its privacy vulnerabilities. In the following, we will survey some existing privacy schemes for wireless networks without network coding, and traditional wireline networks, respectively.

In traditional wireline networks, two fundamental techniques named Mix-Net and Onion Routing are proposed to anonymize end-to-end data communications. Here, schemes based on Chaum's Mix [6], such as Mixminion [7] and MorphMix [8], are termed as Mix-Net. The common feature of them is that they all employ techniques such as shaping, reordering, and layered encryption to eliminate the packet correlations at participating nodes. By layered encryption, the source should successively encrypt each packet with public keys of the nodes along the route. Then, each node peels one layer of encryption with its private key so that the packet finally arrives at the receiver as plain text. On the other hands, Onion Routing refers to a family of anonymity protocols, which are also based on the technique of layered encryption, but are more computationally efficient than Mix-Net. In traditional Onion Routing [9], the source creates a layered structure named onion, by successively encrypting session keys for nodes along the route using their corresponding pubic keys. Then, each node along the route decrypts the onion with its private key to obtain the session key for it. After that, data packets are moved along the route just as in Mix-Nets, except that here packets are symmetrically encrypted by the source with the session keys previously distributed. The technique of Onion Routing is further developed in [10], which ensures forward secrecy for Onion Routing using incremental pathbuilding, and in [11], which eliminates the need of PKI in Onion Routing using multi-path routing. As noted above, both Mix-Net and Onion Routing require relay nodes to perform encryptions/decryptions on packets, and these operations are just in conflict with the packet-mixing operations required by network coding. Thus, neither Mix-Net nor Onion Routing can be applied to networks equipped with network coding.

Then let us examine some typical anonymity techniques designed for wireless networks. Onion Ring [15] is an anonymity scheme proposed for wireless mesh networks. Unfortunately, since Onion Ring is based on Onion Routing, it cannot support network coding, either. ANODR [13] provides an untraceable on-demand routing scheme which can protect user identities in multi-hop ad hoc networks. By using broadcast with tap-door information, ANODR supports distributed route discovery between two arbitrary nodes without revealing sender and/or receiver identities. WAR [14] is another anonymity scheme that exploits the broadcast nature of wireless networks. WAR differs from ANODR in that it has the initiating node select the transmission path, and uses cover traffic to thwart global eavesdropping. However, both ANODR and WAR still need to perform layered encryptions/decryptions on packets, just as in Mix-Net. Thus, they still cannot function when wireless network is upgraded to use network coding.

Different from the above mentioned schemes, Crowds [12] is an anonymity scheme designed for web transactions, and is not based either Mix-Net or Onion Routing. In Crowds, each sender will forward its web requests to a set of random chosen members before the request reaches the web server. Thus, Crowds can incur a considerable delay, and is not suitable for most applications with requirement of realtime communications.

\section{Problem Statement}

\section{A. System Model}

We consider a typical wireless mesh network [3] consisting of wireless routers and clients, as shown in Fig. 2. The routers have minimal mobility and form the infrastructure for clients. Some of these routers along the boundary of the network, termed proxy routers, are responsible for setting up routes for clients directly connected to them. The other routers, termed relay routers, reside at the core of the network and only forward packets along established paths. To enhance data throughput, wireless network coding (i.e., COPE [2]) is enabled in this mesh network: routers operate in promiscuous mode and encode/decode relayed packets opportunistically. We assume that as a basic security guarantee, end-to-end encryption (e.g., SSL/TLS) has already been deployed so that attackers cannot discover the content of packets.

\section{B. Privacy Model}

We now specify the privacy goal we aim to achieve for the system defined above. For a data session that involves com- 


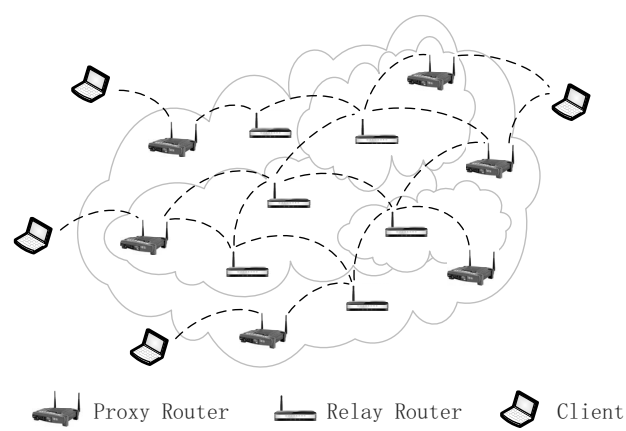

Fig. 2: An example of wireless mesh network.

munication between a sender (i.e., the entity that originates packets) and a receiver (i.e., the entity for which packets are destined), three candidate privacy models given by [16] are considered:

- Communication Unobservability: an adversary cannot distinguish whether a communication exists or not.

- Sender/Receiver Anonymity: an adversary may observe a communication session, but cannot identify the sender/receiver of such a session.

- Relationship Anonymity (or Unlinkability): an adversary may identify a sender or a receiver of some communication, but cannot determine whether they are related or not in the same session.

Note that from the above definitions, communication unobservability offers the strongest privacy guarantee, while the unlinkability offers the weakest among the three. However, in practical systems, unobservability is mainly achieved by injecting dummy/cover packets into networks, which consumes a considerable amount of network bandwidth [22], [23]. Similar performance degradation can be observed in protocols that achieve sender/receiver anonymity. Take Crowds [12] as an example, it provides sender anonymity for web transactions. However, to hide a sender's identity, other nodes in the network need to probabilistically forward the sender's packets to each other. This will incur a large delay since each packet will traverse many more extra hops before reaching the receiver. In short, providing either communication unobservability or sender/receiver anonymity requires significant network resources and may heavily degrade the performance of legitimate applications.

On the other hand, relationship anonymity can be realized with much smaller performance degradation, which is suitable for wireless networks where bandwidth resources are generally more limited as opposed to wireline networks. One successful scheme that achieves relationship anonymity is Onion Routing [9], which is inspired by Chaum's Mix [6]. In general, relationship anonymity is sufficient for most applications that require privacy preservation, since an adversary cannot deduce the sensitive information of "who is talking to whom", even though it can intercept traffic. Thus, to allow for practical deployment, in this paper, we choose to achieve relationship anonymity for the wireless mesh network that we consider.

\section{Adversary Model}

Given the relationship anonymity as the privacy property to preserve, we now define the capabilities of an adversary. The adversary we consider is passive in nature, i.e., it passively monitors network traffic, and will not drop, inject, or modify any packets. The only goal of the adversary is to deduce the information of "who is talking to whom" and establish the sender-receiver relationships of data sessions. To achieve this, the adversary can naively examine some identifiers (e.g., IP addresses) contained in a packet to discover the sender or receiver directly. If such identifiers are protected, the adversary can still perform traffic analysis by content-correlation, sizecorrelation, and time-correlation [5].

In this paper, we will classify the adversary into two categories: (1) the external adversary, which monitors the incoming and outgoing traffic of a target node by staying close to the target node and overhearing packets via the wireless channel; (2) the internal adversary, which compromises and fully controls a target node, and passively analyzes the traffic that traverses the target node. We assume that the proxy routers are trustable, in the sense that they cannot be compromised by internal adversaries. This assumption is reasonable since proxy routers are mostly maintained by local network administrators to provide anonymity service to users belonging to the network, say a LAN. On the other hand, relay routers are placed in the network, and managed by other parties different from the session initiators. Thus, these relay routers rather than the proxy routers have the motivation to compromise users' privacy.

\section{OVERVIEW OF GENERAL FrAMEWORK}

As noted in the current literature, the main task of achieving relationship anonymity is to prevent the adversary from correlating input and output packets of relay nodes. This is commonly achieved using schemes based on Chaum's mix [6], where packets are transformed before being forwarded.

In the following, we first demonstrate the infeasibility of Chaum's mix-based schemes in wireless network coding, and then present the design rationale of our proposed scheme. For clarity of explanation, we adopt the paradigm of Onion Routing [9], a classic mix-based scheme that is the core of many prior anonymous protocols [10], [11], [15]. However, we emphasize that other mix-based schemes can also be used as the building block of our proposed scheme in a similar fashion.

\section{A. Infeasibility of Onion Routing in Network Coding}

Onion Routing [9] is an anonymous routing protocol that can achieve relationship anonymity in traditional networks without network coding. A typical Onion Routing system consists of inter-connected routers called onion routers. Each router $i$ is loaded with a pair of public/private keys $\left(u k_{i}, r k_{i}\right)$, and the global knowledge of the network topology. In the following, we briefly describe how Onion Routing works when no network coding is used, using the simple cross topology shown in Fig. 1(a). 
Suppose that two end users $U_{1}$ and $U_{2}$, who are respectively connected to routers $S_{1}$ and $D_{1}$, want to set up a session. In Onion Routing, $U_{1}$ first sends a connection request to $S_{1}$. On receiving this request, $S_{1}$ determines a path to router $D_{1}$ (in this case, the path is simply $S_{1} \rightarrow C \rightarrow D_{1}$ ). Then $S_{1}$ randomly selects two session keys $s k_{C 1}$ and $s k_{D 1}$ for $C$ and $D_{1}$ respectively, and constructs a layered data structure called an onion as $\left\{\left\{s k_{D 1}, U_{2}\right\}_{u k_{D 1}}, s k_{C 1}, D_{1}\right\}_{u k_{C}}$, where $u k_{C}$ and $u k_{D 1}$ are the public keys of $C$ and $D_{1}$, respectively, and $\{\cdot\}_{k}$ denotes the encryption using public key $k$. Then $S_{1}$ sends this onion to $C$, which uses its private key $r k_{C}$ to decrypt the onion. After decryption, $C$ will obtain its session key $s k_{C 1}$, the next-hop router $D_{1}$, and the embedded onion $\left\{s k_{D 1}, U_{2}\right\}_{u k_{D 1}}$. This embedded onion is then forwarded to $D_{1}$, which decrypts it using its private key to get the session key $s k_{D 1}$. In addition, $D_{1}$ will find that it is the last hop of the route, as the next hop is the end user $U_{2}$ connected to $D_{1}$. Then $D_{1}$ forwards the connection request to $U_{2}$, and a data session is established. After the route establishment, data is transmitted using symmetric-key encryptions. Specifically, using the session keys previously assigned, $S_{1}$ applies symmetrickey encryption to each message $M$ originated from $U_{1}$ and constructs $\left\{\{M\}_{s k_{D 1}}\right\}_{s k_{C 1}}$. Then $C$ removes the outermost layer using $s k_{C 1}$ to get $\{M\}_{s k_{D 1}}$, and finally $D_{1}$ removes the innermost layer using $s k_{D 1}$ to recover message $M$.

Similarly, we can apply Onion Routing for another session that uses path $S_{2} \rightarrow C \rightarrow D_{2}$. We can assign $C$ and $D_{2}$ session keys $s k_{C 2}$ and $s k_{D 2}$ for this session, respectively.

Suppose that network coding is enabled. We now show how Onion Routing fails. First, $D_{1}$ and $D_{2}$ can overhear the packets $\left\{\left\{P_{1}\right\}_{s k_{D 1}}\right\}_{s k_{C 1}}$ and $\left\{\left\{P_{2}\right\}_{s k_{D 2}}\right\}_{s k_{C 2}}$ from $S_{1}$ and $S_{2}$, respectively, and both packets will be received by $C$ as well. Then, $C$ will perform decryption on these two packets and get $\left\{P_{1}\right\}_{s k_{D 1}}$ and $\left\{P_{2}\right\}_{s k_{D 2}}$. By network coding, $C$ would broadcast $\left\{P_{1}\right\}_{s k_{D 1}} \oplus\left\{P_{2}\right\}_{s k_{D 2}}$. However, neither $D_{1}$ nor $D_{2}$ can decode the packets, as they only overhear the packets encrypted with session keys $s k_{C 1}$ and $s k_{C 2}$ possessed by $C$, respectively.

Finally, it is important to note that this simple example provides an illustrative insight for larger topologies. Suppose that an adversary can eavesdrop traffic that traverses node $C$. When Onion Routing is used, the adversary can only tell the previous hops (i.e., $S_{1}$ and $S_{2}$ ) and next hops (i.e., $D_{1}$ and $D_{2}$ ) of node $C$, but cannot determine the nodes that are further upstream or downstream. Such a privacy guarantee cannot be directly achieved with simple end-to-end encryption.

Summary: In wireless network coding (i.e., COPE), a node can use overheard packets of other sessions to decode the packets of its own session. However, when Onion Routing is used, packets are encrypted with different session keys, and nodes cannot perform correct decoding using the stale overheard packets, which are both encoded and encrypted at the same time.

\section{B. Design Rationale of $A N O C$}

Cooperative networking is a relatively new design policy which encourages multiple nodes to cooperate to finish a

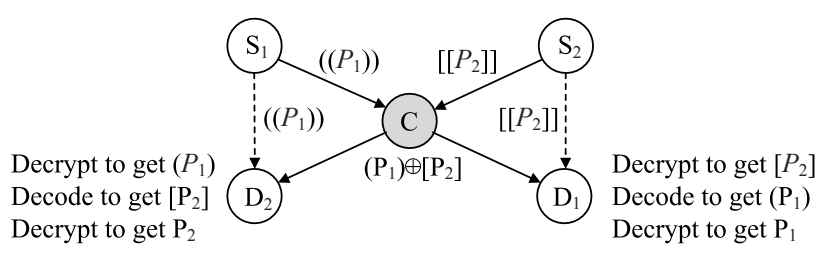

Fig. 3: An illustration of how relay nodes cooperate to make Onion Routing and network coding compatible. (.) and [.] denote the symmetric-key encryptions performed by $S_{1}$ and $S_{2}$ on their data packets, respectively.

common communication goal, and is successfully applied to wireless ad hoc networks [24], [25] and content distribution networks [26]. We observe that the idea of cooperative networking can also be used here to resolve the conflicts between Onion Routing and network coding. Taking the cross topology for example again, the following two-step cooperation (as illustrated in Fig. 3) can help Onion Routing adapt to network coding.

(1) session-key sharing: $C$ shares its session key $s k_{C 1}$ and $s k_{C 2}$ with $D_{2}$ and $D_{1}$, respectively;

(2) auxiliary decrypting: $D_{2}$ decrypts the overheard packet $\left(\left(P_{1}\right)\right)$ using $s k_{C 1}$ to obtain $\left(P_{1}\right)$, and $D_{1}$ decrypts the overheard packet $\left[\left[P_{2}\right]\right]$ using $s k_{C 2}$ to obtain $\left[P_{2}\right]$.

After the above cooperation, $C$ can broadcast the coded packet $\left(P_{1}\right) \oplus\left[P_{2}\right]$. Then, $D_{1}$ can decode this packet to get $\left(P_{1}\right)$, and decrypt $\left(P_{1}\right)$ to get $P_{1}$; similarly, $D_{2}$ can obtain $P_{2}$. In this way, the conflict between network coding and Onion Routing is resolved.

Now, we consider two different approaches to achieve session-key sharing between the coding node $(C$ in the example) and the decoding nodes ( $D_{1}$ and $D_{2}$ in the example). The first but naive approach is to simply let each router share its private key with all its one-hop neighbors (e.g., $C$ shares $r k_{C}$ with $D_{1}$ and $D_{2}$ ), so that when an anonymous session passing through the router is established, each of its neighbors can also obtain the session key. This approach can be carried out during the establishment phase of an anonymous session, and hence will not incur any online overhead. However, the sharing of private keys would severely undermine the security of system.

For the second approach which we are going to adopt, the coding node shares its session keys (instead of private keys) with its one-hop neighbors in an on-demand fashion. Specifically, when there are coding opportunities, the router that performs coding should securely broadcast its session keys of the corresponding sessions to its neighbors. One critical point is that the share of session keys is only limited to neighboring nodes. Nodes that are further upstream or downstream cannot see the session keys; otherwise the user privacy cannot be properly preserved. Another critical point is that the key sharing procedure is only triggered when there are opportunities for network coding, so that session keys will not be shared unnecessarily. 


\section{ANOC: The Details}

We propose ANOC, the anonymous network-coding-based communication for wireless mesh networks. ANOC is built upon the traditional Onion Routing protocol, and introduces efficient cooperation (i.e., session-key sharing and auxiliary decrypting) among relay nodes to resolve the conflict between Onion Routing and network coding. The technical challenges include: (i) how to trigger the session-key sharing in an ondemand fashion, and (ii) how to efficiently and securely share session keys with neighbors without leaking any information to adversaries. In the following, we show how ANOC addresses these two challenges.

Road Map of Illustration. In Subsection V-A, we show how to bootstrap the ANOC protocol in a given wireless mesh network; Subsection V-B describes the formats of packets to be used in ANOC; Subsection V-C shows how to set up an anonymous session with ANOC; Subsection V-D and V-E illustrate how relay nodes cooperate to enable Onion Routing to function with wireless network coding; Subsection V-F specifies how to tear down an existing anonymous session in ANOC.

\section{A. System Setup}

First, each of the routers (including proxy routers and relay routers as shown in Fig. 2) is assigned a unique router identifier and preloaded with a pair of public/private keys. In particular, each proxy router knows about the network topology and the public keys of all other routers in the network; each relay router only knows about its neighboring routers and their public keys. Also, each router maintains a sufficiently large buffer for bathing and reordering packets, such that the time-correlation of its incoming and outgoing packets can be eliminated (see [9] for details).

In the bootstrap stage of ANOC, each router performs operations offline to establish the secure broadcast key and local neighboring table. These operations are explained below.

1) Secure Broadcast Key: Each router $R$ randomly selects its broadcast key, which will be later used for link-layer encryptions of (i) packet headers and (ii) distribution of session-keys. For each of the neighboring routers, $R$ encrypts its broadcast key using the public key of the neighbor and unicasts the ciphertext to that neighboring router. Note that this procedure has a relatively low complexity, since for mesh network consists of $N$ nodes, each of which has an average of $M$ neighbors, only $N \times M$ unicasts are needed. To cope with network dynamics, we also require that: (i) each newly-joined router exchanges its broadcast key with all of its neighbors; and (ii) each router in the network flushes its broadcast keys that are unused for a specific duration.

2) Local Neighboring Table: Each router $R$ maintains a local neighboring table that records the neighbors of each of $R$ 's neighbors. The table will be used to determine whether there are coding opportunities (details will be presented in Section V-D). For instance, for node $C$ in Fig. 1(c), its local neighboring table will specify that node $S_{1}$ has neighbors $S_{2}$ and $S_{4}$. The table can be easily constructed by having each router broadcast the list of its one-hop neighbors.

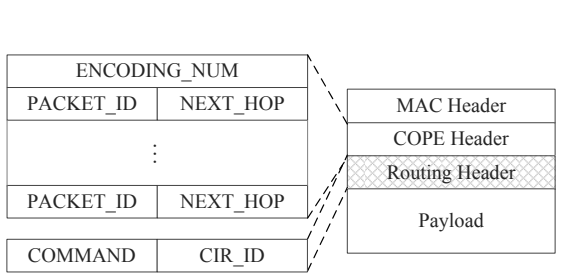

(a) Header Format

\begin{tabular}{|c|c|}
\hline \multicolumn{2}{|c|}{ KEY_NUM } \\
\hline ROUTER_ID & SESSION_KEY \\
\hline CIR_ID_IN & CIR_ID_OUT \\
\hline \multicolumn{2}{|c|}{$\vdots$} \\
\hline ROUTER_ID & SESSION_KEY \\
\hline CIR_ID_IN & CIR_ID_OUT \\
\hline \multicolumn{2}{|c|}{ PADDING } \\
\hline
\end{tabular}

(c) DISTRIBUTE

Packet Payload
ROUTER NUM \begin{tabular}{|l|l|}
\hline SESSION KEY & NEXT HOP \\
\hline
\end{tabular} \begin{tabular}{|l|l|}
\hline SESSION_KEY & NEXT_HOP \\
\hline
\end{tabular} PADDING (b) CONNECT Packet Payload

Fig. 4: The header and payload format of ANOC.

\section{B. Packet Format}

ANOC assumes that wireless network coding (i.e., COPE [2]) is enabled, and the packet header of COPE is placed right after the MAC header. In addition, we add a new routing header to enable anonymous routing. Fig. 4(a) illustrates the layout of the COPE header and the routing header in our protocol. The routing header consists of two fields: COMMAND and CIR_ID. The COMMAND field describes the type of a packet. In ANOC, there are four types of packets:

- CONNECT (Section V-C): for route establishment,

- DISTRIBUTE (Section V-D): for session-key distribution,

- DATA (Section V-E): for information delivery, and

- DESTROY (Section V-F): for tearing down an existing session.

The CIR_ID field carries the circuit identifier which enables multiple sessions to be multiplexed into a single physical channel. We explain its use in Section V-C.

Figs. 4(b)-(d) illustrate the four types of packets (i.e., CONNECT, DISTRIBUTE, DATA, and DESTROY), each of which is attached with different payload fields that are encrypted with different types of keys. We will explain each type of packets and how each is encrypted in the following subsections. In particular, we encrypt the COPE header and the routing header with the broadcast keys that have been established during the bootstrap phase (see Section V-A). Therefore, the sensitive information such as the packet type will not be disclosed to external adversaries. Furthermore, we fix each packet to have the same size using padding so as to prevent an adversary from inferring a session through size correlation [9].

\section{Session Setup}

To setup a new session, the proxy router first selects the path of routers in the network toward the destination. It then generates a CONNECT packet, which specifies the selected routers and the corresponding session keys for each of the 
routers. Each router and its corresponding session key will be encrypted with the public key of the router, such that the CONNECT packet forms an onion structure (refer to Section IV-A).

In addition, when initiating a session, the proxy router randomly chooses a locally unique number (i.e., circuit identifier) to identify the session. This number is placed in the CIR_ID field of the CONNECT packet. When a downstream router receives the CONNECT packet, it will record the circuit identifier in the CIR_ID field, and choose a new circuit identifier and replace the CIR_ID field with it. In this way, each router maintains a circuit-identifier mapping for the session. This mapping will later enable DATA packets to be routed along the path specified in the CONNECT packet.

\section{Session-Key Sharing: The First Step of Cooperation}

In ANOC, session keys are shared in an on-demand manner based on coding opportunities. To discover a coding opportunities, we adopt the flow-based ${ }^{1}$ coding conditions given in [27]:

Packets of two flows $F_{1}$ and $F_{2}$ intersecting at node $C$ can be coded together if:

(a) $D\left(C, F_{1}\right) \in N e i g\left(U\left(C, F_{2}\right)\right)$, or $D\left(C, F_{1}\right)=U\left(C, F_{2}\right)$

(b) $D\left(C, F_{2}\right) \in N e i g\left(U\left(C, F_{1}\right)\right)$, or $D\left(C, F_{2}\right)=U\left(C, F_{1}\right)$ where:

$D\left(C, F_{i}\right)$ denotes the downlink node of $C$ in flow $F_{i}$

$U\left(C, F_{i}\right)$ denotes the uplink node of $C$ in flow $F_{i}$

$N$ eig $(k)$ denotes the set of all neighbors of node $k$

The above coding condition can be generalized to determine whether packets from $n \geq 2$ flows can be coded together, by applying this condition for each pair of flows [27]. We call flows that satisfy this condition to be coding flows, and the node which encodes packets to be coding node. A router can easily judge whether packets of a newly created flow can be coded with existing flows using (i) the local neighboring table (refer to Section V-A), (ii) the identities of uplink and downlink nodes of flows passing through itself, and (iii) the above coding condition. For example, in Fig. 1(a), let $F_{1}=S_{1} \rightarrow C \rightarrow D_{1}$ and $F_{2}=S_{2} \rightarrow C \rightarrow D_{2}$. Since $C$ knows $U\left(C, F_{1}\right)=S_{1}, U\left(C, F_{2}\right)=S_{2}, D\left(C, F_{1}\right)=D_{1}$, and $D\left(C, F_{2}\right)=D_{2}$, it can verify that $D_{1} \in \operatorname{Neig}\left(S_{2}\right)=$ $\left\{D_{1}, C\right\}$, and $D_{2} \in N \operatorname{Nig}\left(S_{1}\right)=\left\{D_{2}, C\right\}$, which implies that $F_{1}$ and $F_{2}$ are two coding flows.

After discovering the coding opportunities, the router can start sharing its session keys. Suppose that there are $n$ coding flows $F_{1}, \ldots, F_{n}$ intersecting at router $C$, then $C$ should distribute its session keys associated with $F_{1}, \ldots, F_{n}$ to all its one-hop neighbors. This is achieved by broadcasting a DISTRIBUTE packet encrypted with $C$ 's broadcast key, which is established in the system setup stage (refer to Section V-A). In addition to the session keys, the DISTRIBUTE packet also contains the incoming and outgoing circuit identifiers, in order that all neighbors of the coding node can properly process overheard packets (see Section V-E). As shown in Fig. 4(c),

${ }^{1}$ Here, a flow is equivalent to a session.

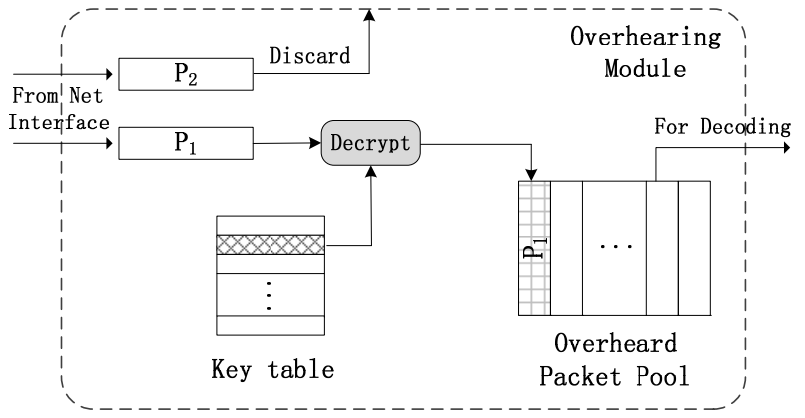

Fig. 5: The overhearing module in ANOC.

a DISTRIBUTE packet contains the tuples (Router_ID, Session_Key, CIR_ID_IN, CIR_ID_OUT), where Router_ID is the identifier of the upstream router of $C$ in this route, Session_key is the session key for $C$ in this route, and CIR_ID_IN and CIR_ID_OUT are the incoming and outgoing circuit identifier for the session, respectively. For instance, let us consider the coding scenario in Fig. 3. Let the mapping of circuit identifiers in node $C$ be $001 \rightarrow 255$ and $102 \rightarrow 123$ for sessions $S_{1} \rightarrow C \rightarrow D_{1}$ and $S_{2} \rightarrow C \rightarrow D_{2}$, respectively, and let $C$ hold the session keys $s k_{C 1}$ and $s k_{C 2}$ for these two sessions, respectively. Then the two tuples contained in the DISTRIBUTE packet would be $\left(S_{1}, s k_{C 1}, 001,255\right)$ and $\left(S_{2}\right.$, $\left.s k_{C 2}, 102,123\right)$.

In our implementation, session keys are distributed incrementally. To illustrate, let us take the scenario in Fig. 1(c). Suppose that there are initially only two anonymous sessions $S_{1} \rightarrow C \rightarrow S_{4}$ and $S_{4} \rightarrow C \rightarrow S_{1}$. According to our protocol, $C$ would distribute its session keys for these two flows to its neighbors. Then $S_{1}, S_{2}, S_{3}, S_{4}$ will all receive and store these keys. Now suppose that a new session $S_{1} \rightarrow C \rightarrow S_{3}$ is created. $C$ then discovers that there are currently three coding flows. $C$ will just distribute its session key for the new session, while the existing two sessions still use the session keys that have just been distributed.

\section{E. Auxiliary Decrypting: The Second Step of Cooperation}

In ANOC, we implement auxiliary decrypting (see Section IV-B) via a separate module named overhearing module, as shown in Fig. 5. This module consists of a key table, an overheard packet pool, and a decrypting unit. The key table stores the tuples (Router_ID, Session_Key, CIR_ID_IN, CIR_ID_OUT) of all DISTRIBUTE packets received from neighbors (see Section V-D). When a router sends a packet, or overhears a packet that is destined to a different MAC address rather than itself, it will find the router identifier of the sender (by mapping to the source MAC address) and the circuit identifier contained in the packet header. It then looks up in the key table for the tuple that has the mapping indexed by (Router_ID, CIR_ID_IN). If no tuple is found, then the overheard packet will be discarded; otherwise, if the tuple is found, then the router will decrypt the overheard packet using the session key in the tuple, and replace the CIR_ID field of the overheard packet with CIR_ID_OUT in the tuple. The resulting packet will be stored in the overheard packet pool. 
When later the router receives a coded packet, it will look up in the overheard packet pool for packets that are necessary for decoding.

\section{F. Session Teardown}

When an initiator needs to tear down one of its sessions, it will send a DESTROY packet along the route. Upon receiving the DESTROY packet, each router en route deletes all the information for the session. The neighboring routers will also expire the session keys for the session (received through DISTRIBUTE packets) after a pre-specified timeout period.

\section{PRIVACY ENHANCEMENT ON ANOC}

In this section, we analytically show how ANOC enhances privacy over a wireless mesh network that enables network coding. We consider the external adversary and the internal adversary in our adversary model defined in Section III-C.

\section{A. The External Adversary}

We argue that ANOC can achieve relationship anonymity against the external adversary, which monitors packets via overhearing the wireless channel and attempts to perform traffic analysis via correlations of content, size, and time. We give our justifications as follows.

(a) Content-correlation. In ANOC, any CONNECT, DATA or DESTROY packet will undergo encryption or decryption when passing through each router. Thus, correlation based on content will be impossible. As for DISTRIBUTE packets, they are encrypted (using the secure broadcast key) and broadcasted without revealing the receiver identities or routing information. (b) Size-correlation. In ANOC, each packet is padded into the same size. Thus, it is impossible to perform traceback by correlating packet sizes.

(c) Time-correlation. With batching and reordering operations performed at each router, a packet cannot be associated with others by examining the sending and receiving time.

\section{B. The Internal Adversary}

In Onion Routing, the whole path of a specific route is known by the involved proxy routers, while the relay routers along the route can only identify their previous and next hops. This means that if one relay router is compromised, then it cannot expose the sender-receiver relationship of the whole route. Clearly, this argument holds in ANOC as well when there are no coding opportunities (as in Onion Routing). On the other hand, when a coding node distributes its session keys using secure broadcast, its one-hop neighbors will inevitably obtain more information. This can allow the internal adversary to discover more hops in addition to its previous and next hops for a given anonymous session. In the following, we show how the internal adversary can leverage the session-key sharing in our ANOC protocol to discover more hops in a session. We then give analytical results to demonstrate the number of additional hops discovered by the internal adversary is rather limited.
Notation. Let $A$ be the router that has been compromised and controlled by the internal adversary, and let $S$ be a session that uses $A$ as a relay router. Let $D_{i}$ (resp. $U_{i}$ ) be the $i$ th downstream (resp. upstream) node of $A$ in session $S$ that is $i$ th hop away from $A$ in the downstream (resp. upstream) direction.

Algorithm. Suppose that there is another session observed at $D_{1}$ that can be coded with session $S$. Then, $D_{1}$ 's session key of $S$ is distributed to its neighbors, according to our ANOC protocol. Algorithm 1 specifies how the adversary $A$ can further deduce $D_{2}$.

\begin{tabular}{l}
\hline Algorithm 1: Extra Downstream Node Discovery \\
\hline Search the Overheard Packet Pool for a packet $P$ that \\
belongs to session $S$; \\
2 Decrypt $P$ using the session key of $D_{1}$ to get $P^{\prime} ;$ \\
3 Calculate the packet identifier (hash) $H\left(P^{\prime}\right)$ of $P^{\prime} ;$ \\
4 Decrypt packets sent by $D_{1}$ using $D_{1}$ 's broadcast key; \\
5 Locate $H\left(P^{\prime}\right)$ in COPE headers of the packets obtained \\
in the last step, and find the corresponding next hop of \\
$D_{1}$ in session $S$ (i.e., $\left.D_{2}\right)$;
\end{tabular}

Remarks. The main idea of Algorithm 1 is that if $D_{1}$ is a coding node, then $A$ can overhear packets of $D_{1}$ (which must be a one-hop neighbor of $A$ ) to determine an extra hop $D_{2}$. Now, given that $A$ knows $D_{2}$, if $D_{2}$ is a coding node and a neighbor of $A$, then $A$ can use the similar procedure to determine $D_{3}$, the next hop of $D_{2}$. To understand this, we consider three cases given in Fig. 6. In Fig. 6(a), $D_{2}$ is a neighbor of $A$, but there is no coding opportunity involving $S$. Then, $S$ cannot obtain the session key of $S$ at $D_{2}$ to deduce $D_{3}$. In Fig. 6(b), $D_{2}$ is a coding node, but $D_{2}$ is not a neighbor of $A$. Thus, $A$ cannot overhear or analyze the packets sent by $D_{2}$ to deduce $D_{3}$. Only in Fig. 6(c), $D_{2}$ is both a coding node and a neighbor of $A$, and $A$ can deduce $D_{3}$ as in Algorithm 1 . In general, if $D_{2}, D_{3}, \cdots, D_{i}$ are coding nodes and neighbors of $A$, then $A$ can discover $D_{3}, D_{4}, \ldots, D_{i+1}$.

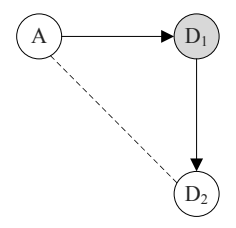

(a)

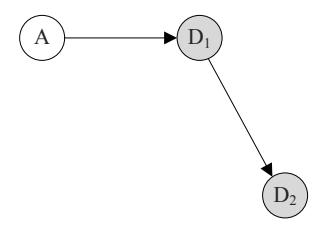

(b)

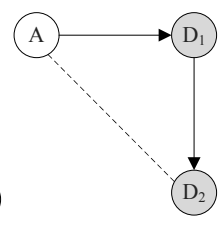

(c)
Fig. 6: Three different cases of $D_{2}$. The dashed line indicates the neighborhood relationship, and a node is shadowed if it has a coding opportunity involving session $S$.

For the upstream direction, $A$ can discover $U_{2}$ in the similar way as in Algorithm 1, i.e., by deducing whether the next hop of $U_{2}$ is $U_{1}$. The only difference is that $U_{2}$ needs to not only have a coding opportunity, but also be a neighbor of $A$ (otherwise, $A$ cannot have the broadcast key of $U_{2}$ to decrypt its packets and analyze its next hop). 
Analysis. We start with the downstream direction, and evaluate how many additional downstream hops (i.e., beyond $D_{1}$ ) that $A$ can identify in session $S$ established using ANOC. From the above discussion, the number of extra hops that can be identified by $A$ depends on the placements of nodes in session $S$ and on the number of coding opportunities of nodes in session $S$. Thus, we define two probabilities for a downstream node $D_{i}$ in session $S$ : (1) $p_{i}$, the probability that node $D_{i}$ has a coding opportunity involving $S$, and (2) $l_{i}$, the probability that node $D_{i}$ is a neighbor of $A$, conditioning on that it has a coding opportunity involving $S$. For simplicity, we would use the unconditional probability $q_{i}=p_{i} l_{i}$ in the following derivations.

The values of the two probabilities $p_{i}$ and $l_{i}$ depend on many factors. Intuitively, for $i \geq 2, l_{i}$ is small (large) in a sparse (dense) network since it is less (more) likely that $D_{i}$ is a neighbor of $A$. One observation is that the probability that a node has a coding opportunity is strictly bounded [4], and the fraction of coding traffic of COPE observed in a randomized setting is below $40 \%$ [27]. Since $q_{i} \leq p_{i}$, we expect that both $p_{i}$ and $q_{i}$ are small in general.

Let $d+1$ be the total number of downstream nodes in session $S$ (i.e., $D_{1}, \cdots, D_{d+1}$ ). The probability that $A$ can determine exactly $n$ additional downstream nodes (beyond $D_{1}$ ) in session $S$ (i.e., $\left.D_{2}, \ldots, D_{n+1}\right)$ is calculated by:

$$
P_{n, S}= \begin{cases}p_{1} \prod_{i=2}^{n} q_{i}\left(1-q_{n+1}\right) & \text { if } 1 \leq n \leq d-1 \\ p_{1} \prod_{i=2}^{n} q_{i} & \text { if } n=d .\end{cases}
$$

For $1 \leq n \leq d-1, P_{n, S}$ is the product of (i) the probability that $D_{1}$ has a coding opportunity, (ii) the probabilities that $D_{i}$ $(2 \leq i \leq n)$ have coding opportunities and are neighbors of $A$, and (iii) the probability that $D_{n+1}$ does not have a coding opportunity or is not a neighbor of $A$ (so $D_{n+2}$ cannot be deduced). For $n=d, P_{n, S}$ is simply the product of (i) and (ii).

To simplify our analysis without losing the main message, we let $p_{i}=p$ and $q_{i}=q$ for all $i$ for some constants $p$ and $q$. Then, the expected number of additional downstream nodes (beyond $D_{1}$ ) that can be identified by the adversary $A$ is

$$
E_{S}=\sum_{n=1}^{d} n P_{n, S}=\frac{p\left(1-q^{d}\right)}{1-q} .
$$

Assume $d \rightarrow \infty$, then we can bound $E_{S}$ by $p /(1-q)$. Actually, $E_{S}$ would converge to this bound fast with the increase of $d$. This can be seen in Fig. 7, which plots the results of Eq. (2) for some values of $p$ and $l$. From Fig. 7, we also observe that for a normal value of $l=0.5, p=0.4$, the expected number of additionally identified hops is less than 0.5 , and even in an uncommon case where $l=0.7, p=0.8$, the expectation will not exceed 2 . This fact justifies the limitation of adversary in discovering additional downstream hops beyond $D_{1}$ in our ANOC protocol.

Similarly we can calculate the expected number of additional upstream nodes that can be identified by the adversary $A$ as (note that each upstream node needs to have a coding opportunity and be a neighbor of $A$, according to our early discussion):

$$
E_{S}^{\prime}=\frac{q\left(1-q^{d}\right)}{1-q}
$$

Note that $E_{S}^{\prime}$ is strictly smaller than $E_{S}$, and thus the expected number of additional upstream nodes identified by $A$ will be less than that of downstream nodes. We are not going to plot the results of Eq. (3), since the curves will be much the same with that of Fig. 7 in shape.

\section{EXPERIMENTAL RESULTS}

We now evaluate ANOC using realistic wireless settings. Our evaluation is based on nsclick [17], which embeds Click Modular Router [28] into the ns2 simulator [29]. A key design feature of nsclick is to enable a routing protocol implemented in Click to be readily deployed in a real networks with minimal configuration changes. Thus, we choose nsclick to ensure that our ANOC implementation mimics an actual system prototype used in practice.

We design two Click modules to reflect our system model: the proxy router module, which defines a proxy router for initiating new sessions and selecting routes, and the relay router module, which defines a relay router for forwarding data packets and performing network coding. For comparisons with ANOC, we also implement the following two routing protocols:

- $C O P E$, the routing protocol with network coding but no anonymity protection,

- Onion, the Onion Routing protocol with anonymity protection but no network coding.

Our experiments focus on the following four metrics:

- throughput, the aggregate throughput of all sessions in the network,

- coding rate, the ratio of the number of encoded packets to the total number of forwarded packets,

- fairness, the measure of how peer flows get equal throughput based on Jain's fairness index [30] $\left(\sum x_{i}\right)^{2} /\left(N \sum x_{i}^{2}\right)$ (where $x_{i}$ denotes the throughput of the $i$ th flow and $N$ is the number of all flows), and

- symmetric-key encryption/decryption, the computational cost of packet processing when anonymity protection is used.

The first three metrics mainly focus on the communication performance, while the last metric evaluates the computation overhead incurred by anonymous routing. In our experiments, we assume that the processing delay of packets due to symmetric-key encryption/decryption is negligible. This is justified by the observation that the communication over the wireless channel is generally the performance bottleneck as opposed to the processing of symmetric-key cryptographic operations $^{2}$.

We carry out experiments using three representative topologies given in Fig. 8: (a) the cross topology, which is relatively simple and serves as the baseline setting, (b) the grid topology, representing a relatively complex but regular setting, and (c) the random topology, a more realistic setting to test the applicability of our scheme.

\footnotetext{
${ }^{2}$ We further conduct a benchmark test with OpenSSL [31] for 192-bit AES (a symmetric-key cryptographic algorithm) on a $2.13 \mathrm{GHz}$ Intel Core $2 \mathrm{CPU}$ and observe that the processing throughput is around $80 \mathrm{MB} / \mathrm{sec}$, which is much higher than the link capacity of an 802.11 wireless channel.
} 


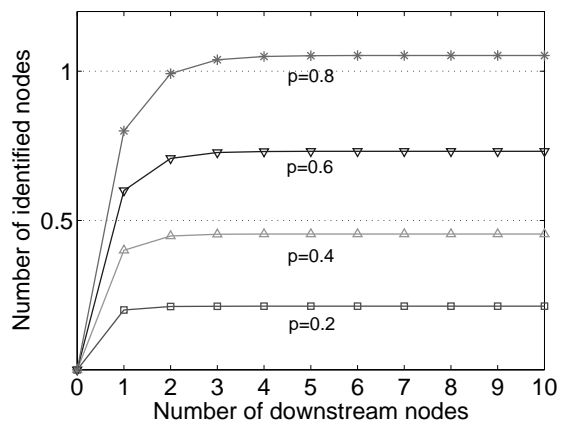

(a) $l=0.3$

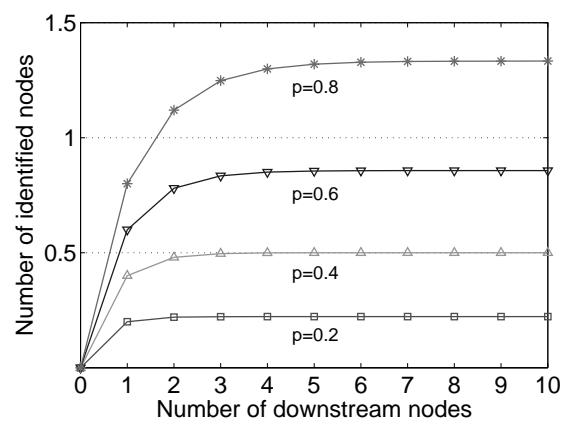

(b) $l=0.5$

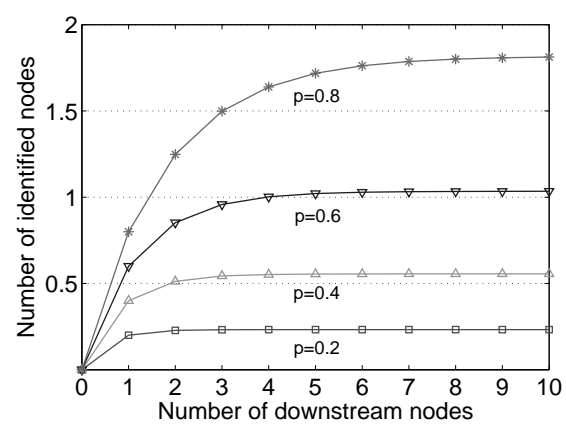

(c) $l=0.7$

Fig. 7: The expected number of additional downstream nodes (beyond $D_{1}$ ) identified by $A$ vs. the total number of downstream nodes (beyond $D_{1}$ ).

Proxy Router

Relay Router

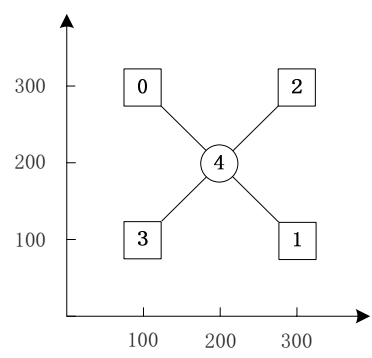

(a) Cross Topology

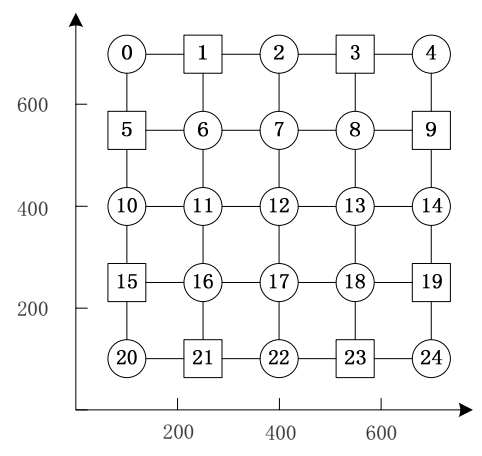

(b) Grid Topology

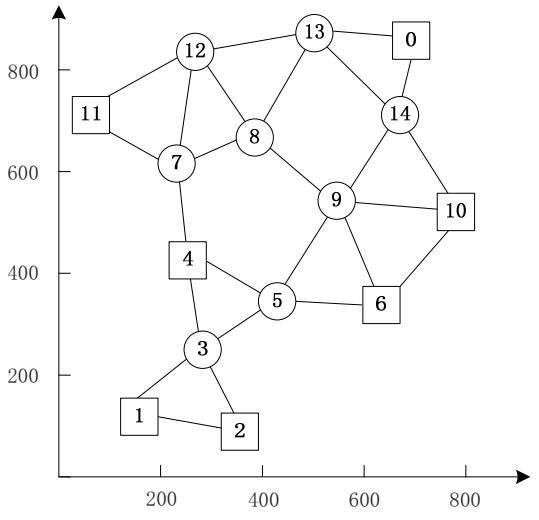

(c) Random Topology

Fig. 8: Three topologies used in our experiments.

Throughout these experiments, we use $802.11 \mathrm{~b}$ and UDP traffic sources with default settings, i.e., the transmission range is set to $250 \mathrm{~m}$ and the carrier sensing range is set to $550 \mathrm{~m}$. For each experiment, we vary the traffic load of participating flows for the collection of results. Note that each data point that we obtain is averaged over 10 simulation runs with different random seeds.

In the following, we will first consider the throughput, coding opportunity, and fairness for each of the topologies with all three routing protocols. Then, we will measure the encryption/decryption performance specifically for Onion and ANOC.

Experiment 1. Cross Topology: We first revisit the simple cross topology (see Fig. 8(a)), in which we create four sessions: $0 \rightarrow 1,1 \rightarrow 0,2 \rightarrow 3$, and $3 \rightarrow 2$, all of which are relayed by router 4 . We assume that these four flows have the same offered load. Fig. 9 shows the performance of different routing protocols. From Fig. 9(a), we observe that when the offered load increases, the aggregate throughput achieved by ANOC also increases and is fairly close to that of COPE, while the throughput of Onion drops. The reason is that when the offered load increases, there is a higher coding opportunity for ANOC and COPE, as confirmed by Fig. 9(b), while ANOC suffers many packet collisions under the high offered traffic load. Also, Fig. 9(c) shows that both ANOC and COPE achieve a high fairness index.

Experiment 2. Grid Topology: We proceed to study the complex but regular grid topology given by Fig. 8(b), in which there are four sessions: $5 \rightarrow 9,15 \rightarrow 19,1 \rightarrow 21$, and $3 \rightarrow 23$. We deploy the 25 nodes in a way that the radio transmission range of each node covers all neighboring nodes along its surrounding square (i.e., a node can have at most eight neighboring nodes). This topology differs from the previous cross topology in that each routing path consists of more than two hops. Fig. 10 shows the obtained results. Similar to Experiment 1, ANOC and COPE have similar performance and they both outperform Onion when the offered load is high.

Experiment 3. Random Topology: We then consider a 15node random topology where nodes are randomly placed over a plane, as shown in Fig. 8(c). We pick five sessions for our evaluation: $0 \rightarrow 10,10 \rightarrow 0,1 \rightarrow 6,4 \rightarrow 2$, and $6 \rightarrow 11$. Fig. 11 shows the results we get, which are mostly consistent with the results in previous experiments. The only difference we want to highlight is that the fairness indices for ANOC and COPE decrease when the offered load increases. The primary reason is due to the asymmetric property of this 


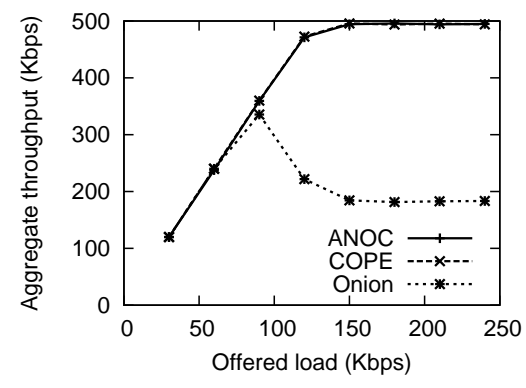

(a) Throughput

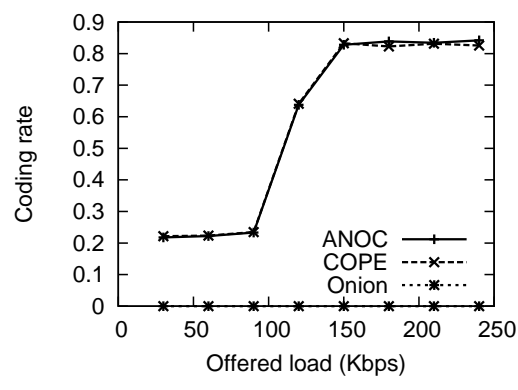

(b) Coding rate

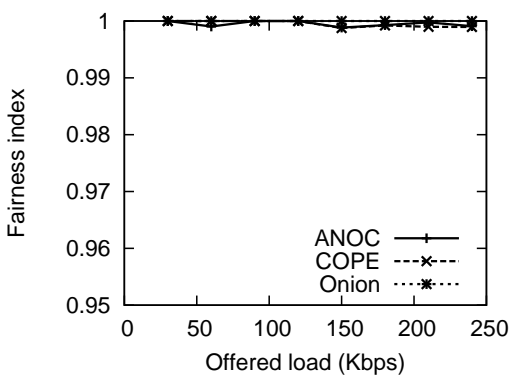

(c) Fairness

Fig. 9: Experiment 1: Throughput, coding rate, and fairness of three protocols in the cross topology.

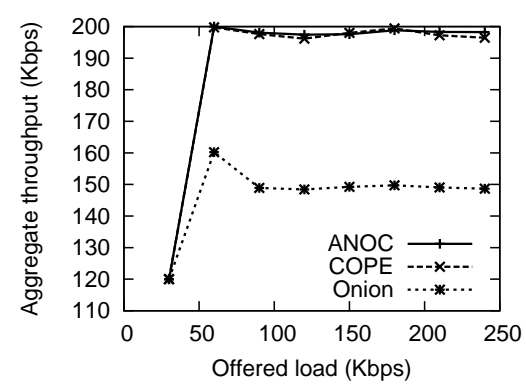

(a) Throughput

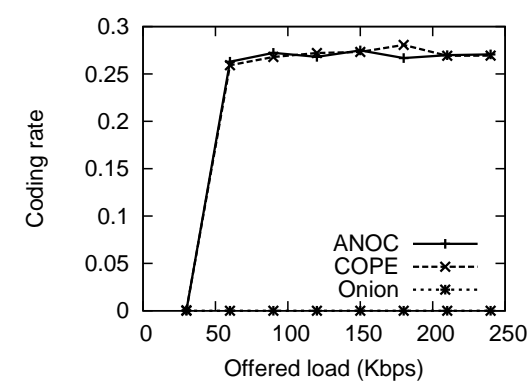

(b) Coding rate

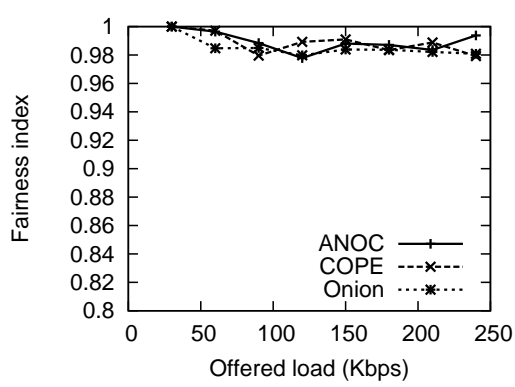

(c) Fairness

Fig. 10: Experiment 2: Throughput, coding rate, and fairness of three protocols for the grid topology.

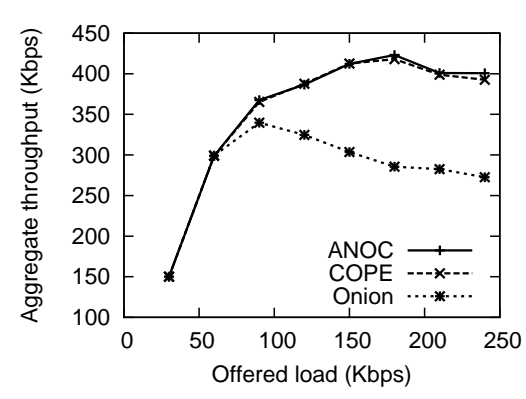

(a) Throughput

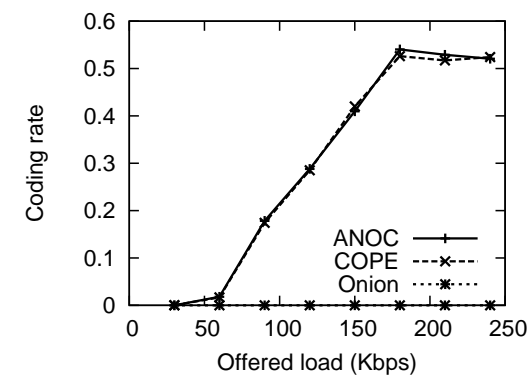

(b) Coding rate

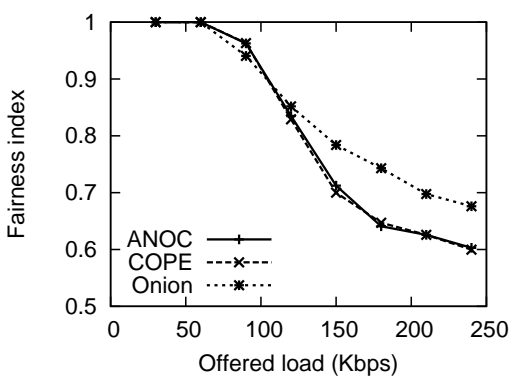

(c) Fairness

Fig. 11: Experiment 3: Throughput, coding rate, and fairness of three protocols for the random topology.

random topology, such that the sessions $0 \rightarrow 10,10 \rightarrow 0$, $1 \rightarrow 6$, and $4 \rightarrow 2$ receive a higher coding opportunity when the offered load increases, while the session $6 \rightarrow 11$ does not. However, this decrease in fairness is independent of the use of anonymous routing.

Experiment 4. Encryption/Decryption: We finally study the performance of encryption/decryption of Onion and ANOC when anonymous routing is used. While we assume the processing delay of encryption/decryption is negligible, it remains interesting to see whether ANOC introduces significantly more encryption/decryption operations on top of network coding. Here, we examine the ratio of the total numbers of symmetrickey encryption and decryption operations to the actual number of packets successfully delivered to the destination, and the results are shown in Fig. 12 and Fig. 13.

We first look at the encryption part. Fig. 12 shows that Onion incurs more encryptions per delivered packet than
ANOC when the offered load increases. The reason is that Onion incurs more packet collisions and hence more lost packets. Encryptions performed on those lost packets will become useless. On the other hand, ANOC takes advantage of the higher coding opportunity with the increased offered load and hence reduces the number of lost packets.

On the other hand, Fig. 13 shows that ANOC incurs more decryptions per delivered packet than Onion, due to the auxiliary decrypting process (see Section IV). For instance, in the grid topology, when a node transmits a packet, up to eight of its one-hop neighboring nodes will overhear the packet and decrypt it. On the other hand, nodes in Onion will not decrypt overheard packets. Thus, we can observe the trade-off when we deploy anonymous routing on top of network coding, as there will be more decryption operations with the use of network coding.

Summary: We show that ANOC, which combines anonymous 


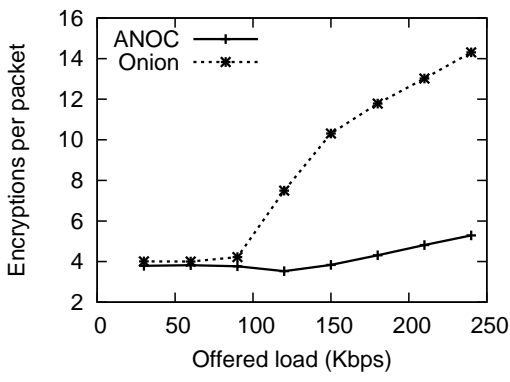

(a) Cross

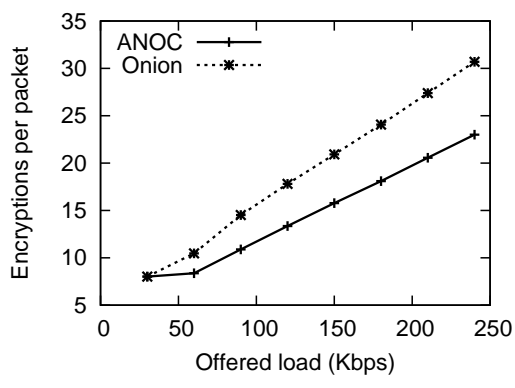

(b) Grid

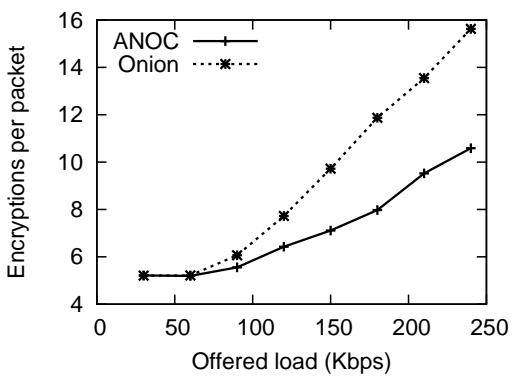

(c) Random

Fig. 12: Experiment 4: The number of encryptions per delivered packet.

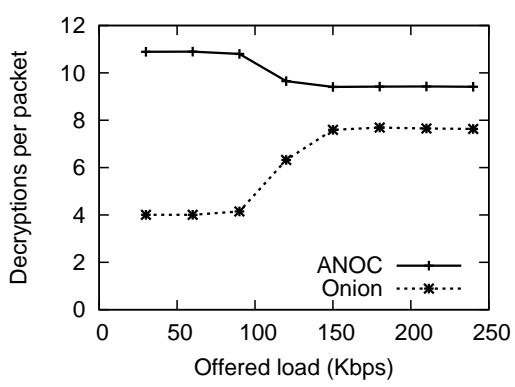

(a) Cross

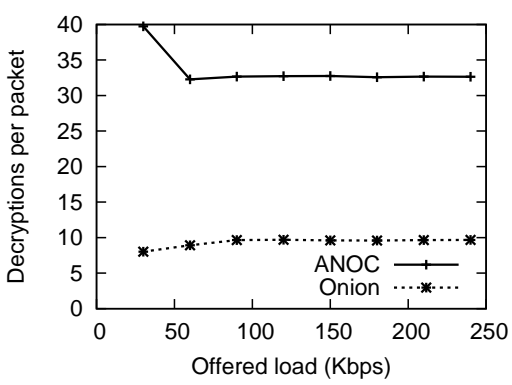

(b) Grid

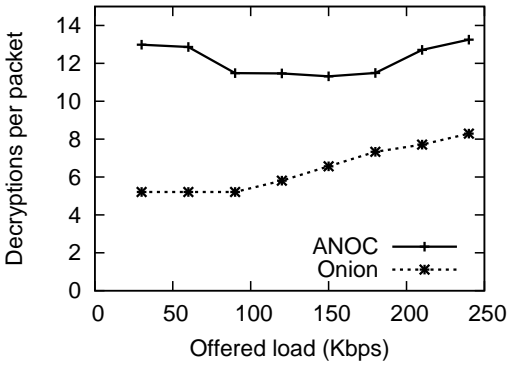

(c) Random

Fig. 13: Experiment 4: The number of decryptions per delivered packet.

routing and network coding, outperforms the traditional Onion Routing protocol (in terms of throughput, coding rate, and fairness), and has similar performance with existing wireless network coding scheme. Thus, ANOC maintains the improvement of the wireless network capacity as in network coding, and in the meantime, provides additional anonymity protection for wireless users.

\section{CONCLUSIONS}

In this paper, we point out an important problem that when a wireless network enables network coding, previously functioning privacy-preserving schemes may no longer perform correctly. To this end, we propose ANOC, a novel anonymous communication protocol which can function seamlessly with wireless network coding. The key technical feature of ANOC is that it uses Onion Routing as the building block, and resolves the conflict between Onion Routing and network coding via efficient cooperation among relay nodes. We present formal analysis to show that the share of session keys in the cooperation procedure will expose a rather limited number of hops to the adversary, and thus relationship anonymity achievable in Onion Routing can be maintained. Furthermore, we perform extensive experiments to demonstrate that ANOC maintains the throughput, coding rate, and fairness as seen in the standard network coding paradigm. This implies that our ANOC not only keeps the advantage of network coding in the effective use of wireless network capacity, but also provides privacy for wireless users at the same time.

\section{REFERENCES}

[1] R. Ahlswede, N. Cai, S.-Y. R. Li, and R. W. Yeung, "Network information flow," IEEE Trans. on Information Theory, vol. 46, no. 4, pp. 1204-1216, Jul. 2000.

[2] S. Katti, H. Rahul, D. Katabi, W. Hu, M. Médard, and J. Crowcroft, "XORs in the air: Practical wireless network coding," IEEE/ACM Trans. on Networking, vol. 16, no. 3, pp. 497-510, Jun. 2008.

[3] I. F. Akyildiz and X. Wang, "Wireless mesh networks: a survey," Computer Networks, vol. 47, no. 4, pp. 445-487, 2005.

[4] J. Le, J. C. S. Lui, and D. M. Chiu, "How many packets can we encode: An analysis of practical wireless network coding," in Proc. of IEEE INFOCOM'08, Apr. 2008.

[5] A. Back, U. Möller, and A. Stiglic, "Traffic analysis attacks and trade-offs in anonymity providing systems," in Proc. of International Workshop on Information Hiding, Apr. 2001.

[6] D. Chaum, "Untraceable electronic mail, return addresses, and digital pseudonyms," Communications of the ACM, vol. 24, no. 2, pp. 84-90, Feb. 1981.

[7] G. Danezis, R. Dingledine, and N. Mathewson, "Mixminion: Design of a Type III anonymous remailer protocol," in Proc. of IEEE Symposium on Security and Privacy, May 2003.

[8] M. Rennhard and B. Plattner, "Introducing MorphMix: Peer-to-Peer based anonymous internet usage with collusion detection," in Proc. of ACM Workshop on Privacy in the Electronic Society, 2002.

[9] M. G. Reed, P. F. Syverson, and D. M. Goldschlag, "Anonymous connections and onion routing," IEEE Journal on Selected Areas in Communications, vol. 16, no. 4, pp. 482-494, May 1998.

[10] R. Dingledine, N. Mathewson, and P. Syverson, "Tor: The secondgeneration onion router," in Proc. of the 13th USENIX Security Symposium, 2004.

[11] S. Katti, J. Cohen, and D. Katabi, "Information slicing: Anonymity using unreliable overlays," in Proc. of NSDI, 2007.

[12] M. K. Reiter and A. D. Rubin, "Crowds: Anonymity for web transactions," ACM Trans. on Information and System Security, vol. 1, no. 1, pp. 66-92, Nov. 1998.

[13] J. Kong and X. Hong, "ANODR: Anonymous on demand routing with untraceable routes for mobile ad-hoc networks," in Proc. of ACM MobiHoc, Jun. 2003. 
[14] M. Blaze, J. Ioannidis, A. D. Keromytis, T. Malkin, and A. Rubin, "Anonymity in wireless broadcast networks," International Journal of Network Security, vol. 8, no. 1, pp. 37-51, Jan. 2009.

[15] X. Wu and N. Li, "Achieving privacy in mesh networks," in Proc. of ACM Workshop on Security of Ad Hoc and Sensor Networks, Oct. 2006.

[16] A. Pfitzmann and M. Hansen, "Anonymity, unlinkability, undetectability, unobservability, pseudonymity, and identity management - a consolidated proposal for terminology," http://dud.inf.tudresden.de/Anon_Terminology.shtml, Feb. 2008, v0.31.

[17] M. Neufeld, G. Schelle, and D. Grunwald, "Nsclick user manual," University of Colorado, Boulder, CO 80309, Tech. Rep., Aug. 2003.

[18] P. Zhang, Y. Jiang, C. Lin, H. Yao, A. Wasef, and X. Shen, "Padding for orthogonality: Efficient subspace authentication for network coding," in Proc. of IEEE INFOCOM, 2011, to appear.

[19] P. Zhang, Y. Jiang, C. Lin, Y. Fan, and X. Shen, "P-Coding: Secure network coding against eavesdropping attacks," in Proc. of IEEE INFOCOM, Mar. 2010.

[20] Y. Fan, Y. Jiang, H. Zhu, and X. Shen, "An efficient privacy-preserving scheme against traffic analysis in network coding," in Proc. of IEEE INFOCOM, Apr. 2009.

[21] Y. Jiang, Y. Fan, X. Shen, and C. Lin, "A self-adaptive probabilistic packet filtering scheme against entropy attacks in network coding," Computer Networks, vol. 53, no. 18, pp. 3089-3101, Dec. 2009.

[22] Y. Yang, M. Shao, S. Zhu, B. Urgaonkar, and G. Cao, "Towards event source unobservability with minimum network traffic in sensor networks," in Proc. of ACM WiSec, Mar. 2008.

[23] M. Shao, Y. Yang, S. Zhu, and G. Cao, "Towards statistically strong source anonymity for sensor networks," in Proc. IEEE INFOCOM, Apr. 2008.

[24] A. Sendonaris, E. Erkip, and R. Aazhang, "User cooperation diversity. Part I. System description," IEEE Transactions on Communications, vol. 51, no. 11, pp. 1927-1938, 2003.

[25] — " "User cooperation diversity. Part II. Implementation aspects and performance analysis," IEEE Transactions on Communications, vol. 51, no. 11, pp. 1939-1948, 2003.

[26] V. N. Padmanabhan, H. J. Wang, P. A. Chou, and K. Sripanidkulchai, "Distributing streaming media content using cooperative networkin," in Proc. of the 12th international workshop on Network and operating systems support for digital audio and video, 2002.

[27] J. Le, J. C. S. Lui, and D. M. Chiu, "DCAR: Distributed coding-aware routing for wireless networks," IEEE Trans. on Mobile Computing, vol. 9, no. 4, Apr. 2010.

[28] E. Kohler, R. Morris, B. Chen, J. Jannotti, and M. F. Kaashoek, "The click modular router,” ACM Trans. on Computer Systems, Aug. 2000.

[29] "The network simulator - ns-2," http://www.isi.edu/nsnam/ns/.

[30] R. Jain, The Art of Computer Systems Performance Analysis. WileyInterscience, 1991.

[31] “The OpenSSL project," http://www.openssl.org/. 